\title{
One Stage Forehead Flap for Nasal Reconstruction
}

\author{
Adel Tolba ${ }^{1}$, Wael Elsh. ${ }^{2}$, Yaser H. $^{2}$, Mansor M. ${ }^{2}$, Hasan $\mathrm{A}^{2}$ \\ ${ }^{1}$ Plastic and Reconstructive Surgery Department, Zagazig Universty Hospital, Zagazig, Egypt \\ ${ }^{2}$ General Surgery Department, Zagazig Universty Hospital, Zagazig, Egypt
}

\section{Email address:}

mansourmorsy@gmail.com (Mansor M.)

\section{To cite this article:}

Adel Tolba, Wael Elsh., Yaser H., Mansor M., Hasan A. One Stage Forehead Flap for Nasal Reconstruction. Journal of Surgery. Vol. 3, No. 6, 2015, pp. 66-70. doi: 10.11648/j.js.20150306.13

\begin{abstract}
Background: The forehead flap is a useful technique to reconstruct deep and large nasal defects. Its disadvantages includes the fact that it is at least a two-stage procedure. The aim of this work is to describe our experience with the use of One stage forehead flap reconstruction to cover nasal defects. Patient: Fifteen patients with extensive nasal defects due to various causes. Full-thickness skin was lost in all cases, $n=7$ cases after excision due to rodent ulcer, $n=5$ cases after squamous cell carcinoma and $n=3$ cases after nasal trauma. Ulcer sizes and sites were demonstrated in table 1 . All were repaired with a single stage forehead flap. The functional and aesthetic results were assessed. Results: High aesthetic and functional goals were achieved in all patients. There were no cases of significant flap necrosis. However, one patient developed mild superficial partial-thickness necrosis. Conclusions: The singe stage forehead flap represents one of the best methods for repair of extensive nasal defects, which may be a substitute for the traditional method (two stages) regard to time repair and cosmetic results.
\end{abstract}

Keywords: Single Stage, Forehead Flap, Nasal Defects

\section{Introduction}

Reconstruction of the nose using distant pedicle flaps has been done for centuries, even before local or general anesthesia existed. Regarding forehead anatomy and its arterial pattern. Forehead flaps are based on the robust vasculature to the forehead via the supraorbital, supratrochlear, and terminal branches of the angular and dorsal nasal vessels. The first anatomic point involves forehead flap terminology. The median forehead flap is harvested from the mid forehead and has a wide pedicle based in the center of the forehead, which originally captured both supratrochlear vessels as seen in the image below. The paramedian forehead flap is designed around a narrower pedicle based on the medial brow area over the superior/medial orbital rim. The skin paddle and pedicle are aligned vertically, with the supratrochlear notch in the paramedian position of the forehead as seen in the image above. The resultant donor scar is oriented vertically and aligns with the medial brow. [1, 2]

The midline forehead flap is a hybrid of median and paramedian flaps, with the skin paddle harvested from the precise center of the forehead. The associated pedicle runs obliquely and is based on a unilateral supratrochlear vessel and collaterals from the medial brow area The pedicle may be based on either side, allowing choices between flap length and the arc of pedicle rotation. ${ }^{2}$

The nose is characterized by several unique qualities, including complex topography, many mobile free margins, adjacent aesthetic subunits, and varying skin properties with respect to thickness, texture, color, and sebaceous content. Nasal defect considerations is necessary for optimal functional and aesthetic surgical reconstruction of the nose. [3]

The supratrochlear artery exits at the superior and medial corner of the bony orbit, approximately at the medial point of the eyebrow. It passes superficial to the corrugator muscle and deep to the orbicularis, ascending in a paramedian position for approximately $2 \mathrm{~cm}$ before piercing the frontalis muscle.

The supratrochlear artery then travels superiorly in the subcutaneous plane, above the galea / frontalis muscle, maintaining numerous anastomoses with the contralateral vessels. The terminal angular artery may ascend the forehead as a distinct vessel or communicate with the ipsilateral supratrochlear artery. The paired dorsal nasal arteries usually merge to form a single central artery of the forehead. [3, 4, 5]

Because of the ideal quality of its color and texture, forehead skin has been acknowledged as the best donor site with which, the forehead is composed of skin, subcutaneous 
fat, frontalis muscle, and a thin layer of areolar tissue that overlies the periosteum and bone.

The aim of this study is to evaluate the nasal reconstruction through one stage operation in a series of fifteen patients depending on the supra-trochlear artery.

\section{Patients and Methods}

This prospective study done in the reconstructive unit of surgery department, the period between 2011 to 2013.

In this type of forehead flap after excision of nasal skin tumor, design of the paramedian forehead flap based on supra-trochlear vessels was done It is necessary to design location of the island flap; so that flap pedicle can easily rotate 180 degrees subcutaneously and reaches to the nasal defect without tension. Furthermore, we must determine the correct location of pedicle with Doppler probe and should determine design of skin along this artery; then release and preparation of the flap from distal to proximal was done. (Fig $1,2)$.

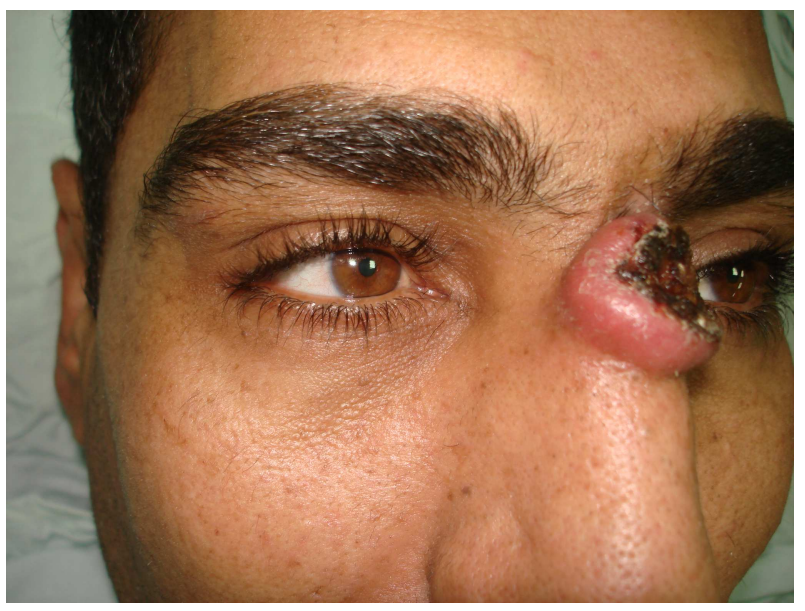

Figure 1. Basal cell carcinoma in a male patient, 33 years old.

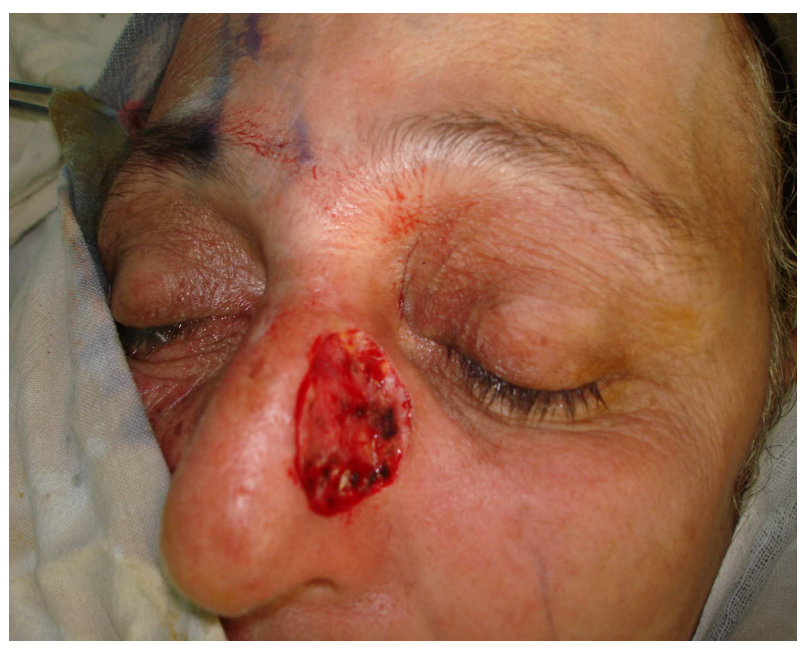

Figure 2. Recurrent basal cell carcinoma.

There was no skin graft necessity in the donor site of the island paramedian forehead flap. After operation, we had some swelling and venous congestion in the subcutaneous plane in the glabella but with a period of time, the swelling reduced; also distance between two eyebrows preserved without any unusualness in contour or location of eyebrows.

Table 1. Preoperative patients characters.

\begin{tabular}{|c|c|c|}
\hline \multicolumn{3}{|c|}{ Preoperative patients characters } \\
\hline \multicolumn{3}{|l|}{ Age (y) } \\
\hline & Range $25-55$ & \\
\hline & Mean age $33 \pm 3.2$ & \\
\hline Sex; & $\mathrm{M} / \mathrm{F} 15 / 5$ & \\
\hline \multicolumn{3}{|c|}{ Causes of the defects; } \\
\hline & Basal cell carcinoma & $\mathrm{n}=(10)$ \\
\hline & Squamous cell carcinoma & $\mathrm{n}=(2)$ \\
\hline & Trauma & $\mathrm{n}=(3)$ \\
\hline \multirow[t]{2}{*}{ Defect sizes ; } & Range 1.5 to $3 \mathrm{~cm}$ & \\
\hline & Median $2.3 \mathrm{~cm}$ & \\
\hline \multicolumn{3}{|c|}{ Sites of the defect } \\
\hline & Ala of the nose (3) & \\
\hline & Nasal tip (1) & \\
\hline & Side wall (6) & \\
\hline & Dorsum (5) & \\
\hline
\end{tabular}

Preoperative patients characters showing the age, sex and site of the lesion

\section{Surgical Technique}

Thorough preoperative planning, including assessment of the defect, hairline height, and forehead laxity, and patients consent about the final outcome of their nasal reconstruction. The operation were done for all patients under general anesthesia A broad-spectrum prophylactic antibiotic was taken one hour before surgery

Placing the patient in the supine position in approximately $20^{\circ}$ to $30^{\circ}$ of the reverse Trendelenburg position has the advantage of decreasing intraoperative bleeding by preventing venous pooling and flap congestion. an intravenous sedation, consisting of a narcotic, such as fentanyl citrate, and a propofol drip. They are then locally anesthetized using 1\% lidocaine with a 1:100,000 concentration of epinephrine. Fig 3

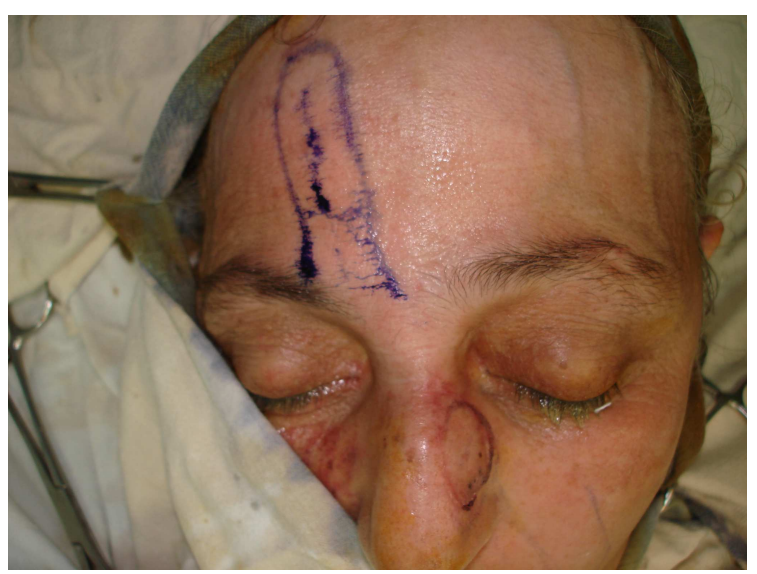

Figure 3. Marking the supra-orpital pedicle flap.

A template of the corresponding nasal subunit, containing the defect, is made from surgical foam or from the foil of a suture pack. The flap can be designed using a Doppler probe 
to precisely locate the supratrochlear artery as it exits the superior medial orbit. This will allow narrowing of the pedicle to as little as $1.0 \mathrm{~cm}$ at the glabella. The flap is then precisely outlined on the forehead with a surgical marker as close to midline as the pedicle will allow.

The flap is then elevated along a cleavage plane superficial to the periosteum of the frontal bone. The flap is elevated from superior to inferior, with care being taken as the dissection reaches 1 to $2 \mathrm{~cm}$ above the eyebrow. Blunt dissection is then performed to avoid cutting the supratrochlear artery as it exits over the corrugator supercilii muscle. Once the artery has been identified and preserved, blunt dissection and sharp dissection are used to continue the dissection down to the root of the nose to achieve adequate flap length. Once the flap is elevated, it is pivoted $180^{\circ}$ on its base. Fig 4,5,6

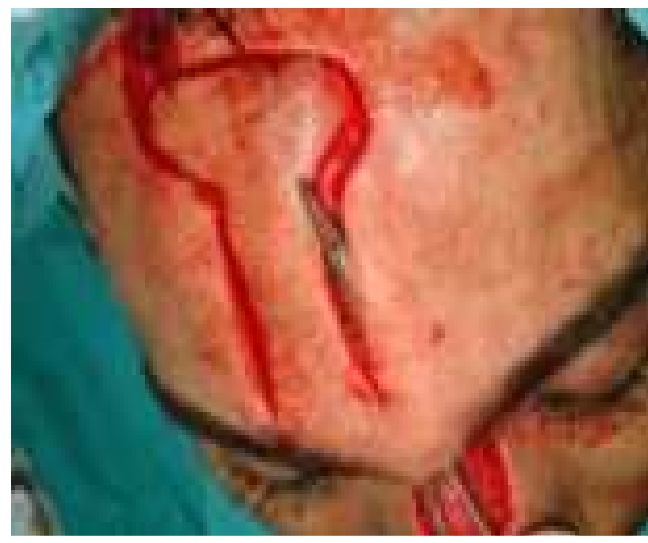

Figure 4. Incision marking and tunneling of the supra-orpital flap.

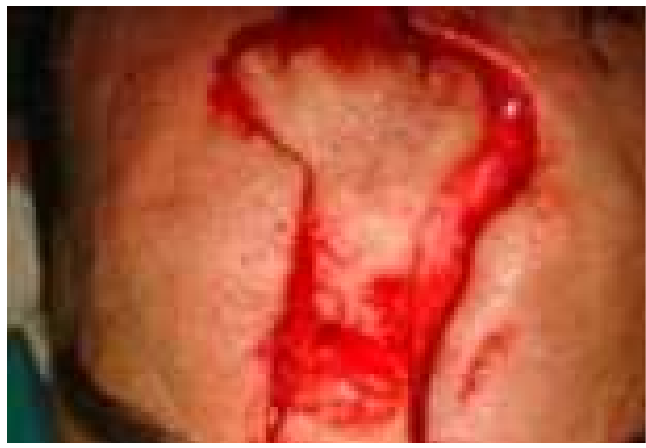

Figure 5. De-epithelialization of the proximal skin in a male patient.

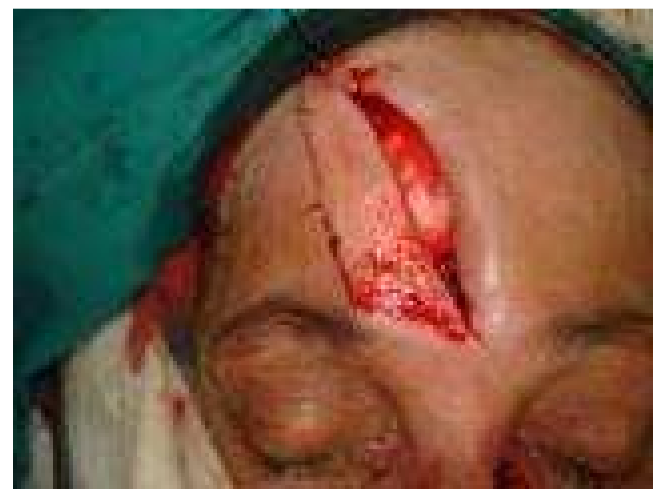

Figure 6. De-epithelialization of the proximal skin in a female patient.
De-epithelialization and thininng of the flap pedicle proximally in the distance which had imbedded under nasal bridge during its rotation debulking is relatively safe in non smokers, non diabetics and middle age because of the flap's rich blood supply.

Our modification involves removal of radix and proximal nasal skin and fat and deepithelialization of the proximal pedicle to allow inset without excess compression or kinking. This modification safely provides acceptable results and avoids a mandatory secondary procedure. Fig 7,8

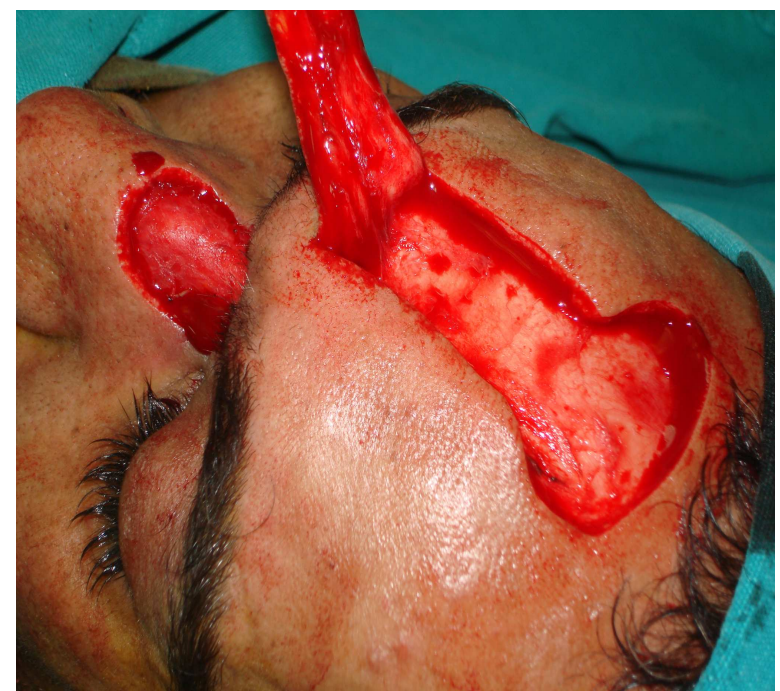

Figure 7. Elevation of the flap.

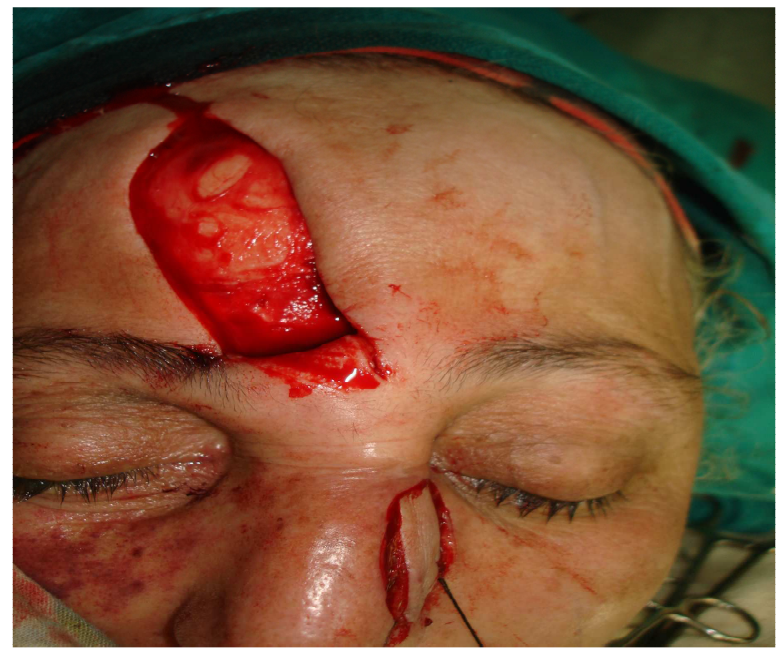

Figure 8. Tunnling of the forehead flap.

Patients were discharged home after 24 hours for observation and wound care. and to ensure adequate intravenous hydration. Prescriptions at discharge include a broad-spectrum antibiotic, for 7 to 10 days. A mild pain reliever, such as acetaminophen

Deep closure is not necessary, since the wound should be under no tension. The skin is closed with a 5-0 or 6-0 polypropylene suture. intraoperative immediate tissue expansion with a towel clamp for few minutes used for wound edges approximations, Fig 9,10 


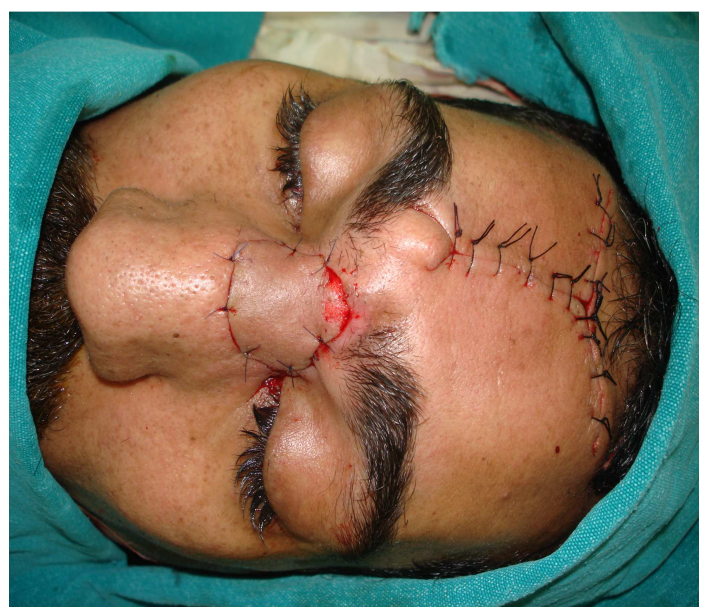

Figure 9. Immediate postoperative picture after tunneling and fixation.

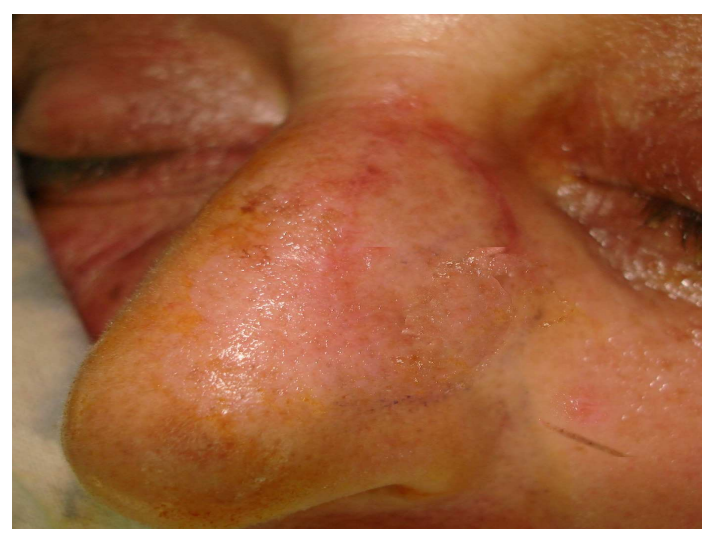

Figure 10. Delayed postoperative picture 6 months ago.

\section{Results}

The patients ages included in the present study, were ranged between 25 to 55 years old with a mean age of 33 years, $n=$ five female and $n=$ ten male patients. The nasal defects had been repaired using the forehead flap after wide excision of basal cell carcinoma $(n=10)$ or squamous cell carcinoma $(n=2)$ and skin trauma in $(n=3)$ cases.

Defect sizes ranged from 1.5 to $3 \mathrm{~cm}$ (median, 2. $3 \mathrm{~cm}$ ). While multiple nasal sites were involved with defects, the nasal tip in $n=1$ patients, on the ala in $n=3$ patients, on the sidewall in $n=6$ patients.

The nutrient vessel in all flaps was supratrochlear artery. The average follow up time was 10 months (3-12 months), and no recurrence occurred. All patients were non-smoker and non-diabetic. High aesthetic and functional goals were achieved in all patients.(fig 10)

No tumor recurrences or significant episodes of local bleeding or infection occurred in this population. There were no cases of significant flap necrosis. However, 2 patients (15\%) developed mild superficial partial-thickness necrosis, which was thought to be related to a combination of length, thinning, and cautery for follicular destruction for columella reconstruction in one patient and to flap extension way past midline for an extensive defect in the other. Both healed uneventfully simply with granulation.
Potential complications with the use of the forehead flap for nasal reconstruction, which are similar to those involved in any flap reconstructive procedure, include bleeding, poor scarring, infection, dehiscence, distortion of free margins, and flap necrosis

Table 2. Postoperative patients complications.

\begin{tabular}{lll}
\hline Postoperative comp. & No & $\%$ \\
\hline Wound Infection & 1 & $(6.5 \%)$ \\
Flap ischemia & 0 & \\
Tip flap necrosis & 2 & $(13 \%)$ \\
Bleeding(intraoperative) & 4 & $(26 \%)$ \\
Patients satisfaction & & \\
Fair & $2(13 \%)$ & \\
Good & $13(85 \%)$ & \\
\hline
\end{tabular}

Postoperative patients complications showing wound infection and partial tip flap necrosis, bleeding with good satisfaction results.

\section{Discussion}

The forehead is multilamellar, consisting of skin, subcutaneous tissue, frontalis muscle, and a thin, areolar layer. as a full- thickness flap based on a paramedian pedicle, its supratrochlear vessels pass deeply over the periosteum at the supraorbital rim and travel vertically upward through the muscle to lie at an almost subdermal position under the skin at the hairline. It is both a myofascial and axial flap, and highly vascular. Excision of the frontalis muscle and subcutaneous fat at the time of initial transfer removes the myocutaneous component of its blood supply [7]

For many years nasal reconstruction was occurred through the pedicled forehead flap until the publication of Reece who used supra-trochlear artery as the axial blood supply to the paramedian forehead flap ${ }^{[7]}$

Forehead flap is said to support random flaps five times the length of the base.[8]some authors fear of ischemia in diabetic and smoker patients We dissected flap pedicle in a tunnel portion sub-periosteal for capture of deep periosteal branch of supra-trochlear artery. Periosteal branch of artery extended beyond $3 \mathrm{~cm}$ above the supraorbital rim and sends additional perforators into the flap. [7]

The goals of nasal reconstruction are the restoration of an esthetic, functional nose with minimization of the donor site deformity ${ }^{[9]}$

We advised using skin graft from the donor region around the nasal defect but in large nasal defects, reconstruction with local flaps, has an advantage that it can be accomplished in one stage but as in any reconstruction, we must consider esthetic results. As it provide excellent color and texture match to avoid two stage operations.

Okada and Maruyama reported a forehead flap based on the wide subcutaneous pedicle, including bilateral supraorbital and supratrochlear vessels, as compared with them in our study, the pedicle of the this flap was much longer and narrower less than $1 \mathrm{~cm}$ wide, including only unilateral supratrochlear vessels. [10] 
Kilinic and Bilen [11] reported one stage reconstruction island flap with a maximum size of $6 \times 7 \mathrm{~cm}$. they stated that the supratrochlear artery was found to consistently exit the superior medial orbit approximately 1.7 to $2.2 \mathrm{~cm}$ lateral to the midline, and continued its course vertically in a paramedian position approximately $2.0 \mathrm{~cm}$ lateral to the midline. In this course we exposed the artery during planning of our flap, and venous congestion was avoided because of the wide tunneling dissection not less than 1.5 $\mathrm{cm}$ versus other authors who dissected the artery subperiosteally and diameter of the tunnel that was dissected two times of pedicle with, also we tried to minimize contour problem and glabella fullness, through the resection of procerous muscle. [7]

Shumrick and Smith 2 demonstrated that the end arterioles of the supratrochlear vessels travel superficial to the frontalis muscle in the upper third of the flap, this allowed us to thin the flap considerably to and Thinning the flap greatly decreases the need for revision surgery to debulk the flap[12]

Quetz and Ambrosch published three stages of forehead flap for nasal reconstruction in difficult cases, the aim is to avoid the need of skin graft or other flap technique as the best tissue color and skin texture is forehead flap with good results and patients satisfactions [13]

\section{Conclusion}

The single forehead flap represents one of the best methods for repair of nasal defects. Outstanding functional and cosmetic results can be achieved. This modification avoids the sequelae of an external pedicle, which include bleeding, dressings, and the patient's reluctance to appear in public.

\section{References}

[1] Menick FJ. Nasal reconstruction: forehead flap. Plast Reconstr Surg 2004; 113(6):100 -111.
[2] Kleintjes WG. Forehead anatomy: arterial variations and venous link of the midline forehead flap. J Plast Reconstr Aesthet Surg. 2007; 60(6):593-606. Epub 2007 Feb 6.

[3] Johnson TM Baker SR Swanson N .Concepts of sliding and lifting tissue movement in flap reconstruction. Dermatol Surg. 2000; 26274- 278.

[4] Tollefson TT, Kriet JD. Comple nasal defects. In Park SS, ed. Facial Plastic Surgery Clinics of North America. Philadelphia, PA: Elsevier Inc; 2005.

[5] Woodard CR, Park SS. Reconstruction of nasal defects $1.5 \mathrm{~cm}$ or smaller. Arch Facial Plast Surg. Mar-Apr 2011; 13(2):97102.

[6] Kheradm and Motamedi MH Nasal reconstruction: experience using tissue expansion and forehead flap. J Oral Maxillofac Surg 2011; 69:1478-84.

[7] Reece EM, Schaverien M, Rohrich RJ. The paramedian forehead flap: a dynamic anatomical vascular study verifying safety and clinical implications. Plast Reconstr Surg 2008; 121:1956-63.

[8] McGregor IA, Morgan G. Axial and random pattern flaps. Br J Plast Surg 1973; 26:202-13.

[9] Zuker RM, Capek L, de Haas W. The expanded forehead scalping flap: a new method of total nasal reconstruction. Plast Reconstr Surg 1996; 98:155-9.

[10] Okada E, Maruyama Y. A simple method for forehead unit reconstruction. Plast Reconstr Surg 2000; 106:111-4.

[11] Kilinc H, Bilen BT. Supraorbital artery island flap for periorbital defects. J Craniofac Surg 2007; 18: 1114-9.

[12] Shumrick KASmith TL The anatomic basis for the design of forehead flaps in nasal reconstruction. Arch Otolaryngol Head Neck Surg. 1992; 118373- 379.

[13] Quetz J, Ambrosch P. Total nasal reconstruction: a 6-year experience with the three-stage forehead flap combined with the septal pivot flap. Facial Plast Surg 2011; 27:266-75. 